\title{
The Human Touch
}

\section{The more I rely on software and programmed/ programmable devices, the more I find I need help from a knowledgeable and timely reachable person. FAQs and YouTube videos}

are often very helpful but when you get trapped into a situation where you have lost your ability to reach an online service, or your smartphone has become a useless brick, or your operating system has decided it hates you, you really want expert help fast. These things never happen at a convenient time. It's the holiday weekend, or 5 P.M. Friday, or 3 A.M. when you are pulling an all-nighter to get a big and important piece of work done. You do not want to hear "call us during office hours" or "we don't have a help service, see our FAQ."

As the global Internet allows vendors or equipment or online application providers to serve a global market, it becomes increasingly important to the globally distributed user population to be able to get assistance when it is needed. In some cases, self-service works very well. For example, overnight shippers often have a "where's my stuff" online help Web page that can tell you where your stuff was last seen (not where it $i s$, necessarily). When you need contextual tutorial assistance, however, these methods may prove inadequate. Apple has made impressive strides with its Apple stores and "genius" assistants-and consumers seem to value consultation services enough to actually pay for them. One may have to schedule an appointment and appear in person, but for some situations, this may be the most effective way to treat such matters as configuration, equipment, or update problems.

Artificial intelligence may pave the way for some forms of local and timely help although I have my share of stories with Siri and Google speech recognition systems, just for example, where they seem to misunderstand queries and commands deliberately. I recall one instance in which a map assistant persistently understood the address: "1312 Maple Street" as "131 to Maple Street" at which point it said it did not understand what I was asking. Recently, I was trying to find a restaurant in New York City, "Le Bernardin" and got "where is the burner diner in New York City?" No variation that I could find worked, including trying to spell the restaurant's name. Mispronouncing "lay Bernard inn" produced "The Barnard Inn" in Vermont. After a few more failures, you may imagine what I wanted to say to this otherwise helpful agent.

In many ways, I am optimistic that speech recognition will get better, especially as context and semantics are incorporated more deeply into the recognition systems. Yet there still seem to be a great many cases where human knowledge and understanding are vitally needed to solve customer problems. One thing that has become helpful is the ability to place phone calls or to open chat windows in context of a particular application. The ability to share the screen to show a customer service agent what you are seeing is also important. This morning, I ran into a problem with a payment system that required input in a particular field but refused to accept any of the choices it offered me to type in. I was stuck and so was the live chat room customer service representative who was unable to reproduce the re- sults. A few hours later, I received an email message saying this was a new service and a bug had been found that caused the recalcitrant program to reject anything I entered for that field. It was gratifying to receive the feedback in a timely way.

As configuration complexity and scale grow, the need for smarter configuration systems, better online assistance, and the ability to share context with customer service agents will become increasingly important. It also seems as if the ability to export state information for later analysis or to inform customer service representatives of the current user's state will become valuable. However, this kind of access may also raise privacy and/or security concerns. Plainly, the computer science community has a lot to contribute, if it can figure out how to overcome these challenges.

While speaking of challenges and the human touch, it seems equally timely and relevant mention to the upcoming 25th Anniversary of the Americans with Disabilities Act on July 26, 2015. Making software that adapts to user needs is a big challenge but it is a vital objective. There are almost certainly laws like this in other countries where an effort has been or is being made to fashion adaptable user interfaces and experience to meet the assistive needs of many users of products and software services.

Vinton G. Cerf is vice president and Chief Internet Evangelist at Google. He served as ACM president from 2012-2014.

Copyright held by author. 